\title{
ELECTROCHEMICAL BEHAVIOUR OF COPPER IN POTASSIUM THIOCYANATE SOLUTION-II. ANALYSIS OF POTENTIOSTATIC CURRENT TRANSIENTS
}

\author{
M. G. Figueroa,* M. F. L. De Mele, R. C. Salvarezza and A. J. Arvia
}

Instituto de Investigaciones Fisicoquímicas Teóricas y Aplicadas (INIFTA), Facultad de Ciencias Exactas, Universidad Nacional de La Plata, Casilla de Correo 16, Sucursal 4, (1900) La Plata, Argentina

(Received 20 January 1986; in revised form 7 April 1986)

\begin{abstract}
The kinetics of passivity and pitting corrosion of copper in borate-boric acid buffer containing potassium thiocyanate is studied at $25^{\circ} \mathrm{C}$ by means of potentiostatic transients recordcd at different constant potential values. Three different potential regions are distinguished. Region $I$, as the applied potential is increased, implies the successive formation of cuprous thiocyanate, cuprous and cupric oxide. This corresponds to the formation of a composite anodic layer. Region II extends from the potential of the third anodic voltammetric peak up to the breakdown potential. Region III involves the breakdown of the passive layer caused by thiocyanate ions, and the formation of different copper salts in the pits. The kinetics of the various stages is discussed in terms of an extension of the nucleation and growth mechanism following an approach which was earlier applied for explaining the initiation and growth of pitting corrosion of metals.
\end{abstract}

\section{INTRODUCTION}

The influence of anions in the electrochemical behaviour of copper in nearly neutral buffered and alkaline solutions has been studied over a wide range of experimental conditions [1-3]; generally implying an electrode surface initially covered by a protective layer consisting of an inner $\mathrm{Cu}_{2} \mathrm{O}$ layer and an outer CuO hydrated layer, as revealed by ESCA and ISS [4]. When aggressive anions such as chloride, bromide or iodide are present in solution the passive film loses its protective characteristics leading to breakdown and pitting corrosion. This process starts when the applied potential exceeds a certain critical value $\left(E_{b}\right)[5]$.

In part $I$, the effect of thiocyanate ions on the electrodissolution and passivation of copper was reported[6]. Accordingly, when the concentration of potassium thiocyanate is close to $0.1 \mathrm{M}$, the electrode surface was covered by a thick layer of CuSCN but as the potential is gradually increased an inner layer made of $\mathrm{Cu}_{2} \mathrm{O}$ and hydrated $\mathrm{CuO}$ was detected so that a complex film structure is accomplished. However, film breakdown and pitting corrosion appears at potentials more positive than $E_{b}$.

The present paper refers to the analysis of potentiostatic current transients recorded in different potential regions of the polarization curve to obtain information on the kinetics of passive film formation and pitting corrosion of copper in borate-boric acid buffer containing potassium thiocyanate. The kinetics of the various stages related to pitting corrosion is interpreted through the nucleation and growth theory following an approach similar to that already discussed for the initiation and growth of pitting corrosion of metals and alloys in the presence of different aggressive anions [7-9].

* Present address: Facultad de Química, Potificia Universidad Católica, Santiago de Chile, Chile.

\section{EXPERIMENTAL}

Working electrodes were made of polycrystalline 99.9 copper rods ( $3 \mathrm{~mm}$ dia.) axially embedded in Araldite holders to obtain circular exposed areas of $0.07 \mathrm{~cm}^{2}$. Firstly the metal surface was mechanically polished with fine grained emery paper, then with alumina paste $0.3 \mu \mathrm{m}$ dia. on polishing cloth. Measurements were made in a conventional Pyrex glass cell containing 0.21 of solution at $25^{\circ} \mathrm{C}$. The potential of the working electrode was measured against a saturated calomel electrode (sce) provided with a Luggin-Haber capillary tip. A platinum plate was used as counter electrode. The electrolyte solution consisted of $0.15 \mathrm{M} \mathrm{H} \mathrm{H}_{3} \mathrm{BO}_{3}+0.075 \mathrm{M} \mathrm{Na} \mathrm{Na}_{2} \mathrm{~B}_{4} \mathrm{O}_{7}$ containing $x$ M KSCN $(0<x<1 \mathrm{M})$. Singular triangular potential voltammograms and potentiostatic current transients were recorded in the conventional way. The rotating ring disc electrode technique (rrde) (gold ring $0.030 \mathrm{~cm}^{2}$ geometric area, copper disc 0.125 $\mathrm{cm}^{2}$ geometric area) was also employed to detect soluble $\mathrm{Cu}(\mathrm{I})$ and $\mathrm{Cu}(\mathrm{II})$ species. A more detailed description of the experimental methods was given in part I[6].

\section{RESULTS}

\section{Potentiodynamic polarization curves}

Potentiodynamic polarization curves run at 0.002 $\mathrm{V} \mathrm{s}^{-1}$ covering the $-1.0 \mathrm{~V}$ to $0.6 \mathrm{~V}$ range in borax buffer containing $0.3 \mathrm{M}$ potassium thiocyanate (Fig. 1) exhibit three different regions. Region I corresponds to various anodic processes, namely, the formation of an inner $\mathrm{Cu}_{2} \mathrm{O}$ layer $(\mathrm{AII}$, AlI) [6], the formation of a hydrous outer CuO layer (AIII, AIII') and the appearance of a cuprous thiocyanate solid phase (AI). Region II covers the passivity range where the current increases according to the potassium thiocyanate con- 


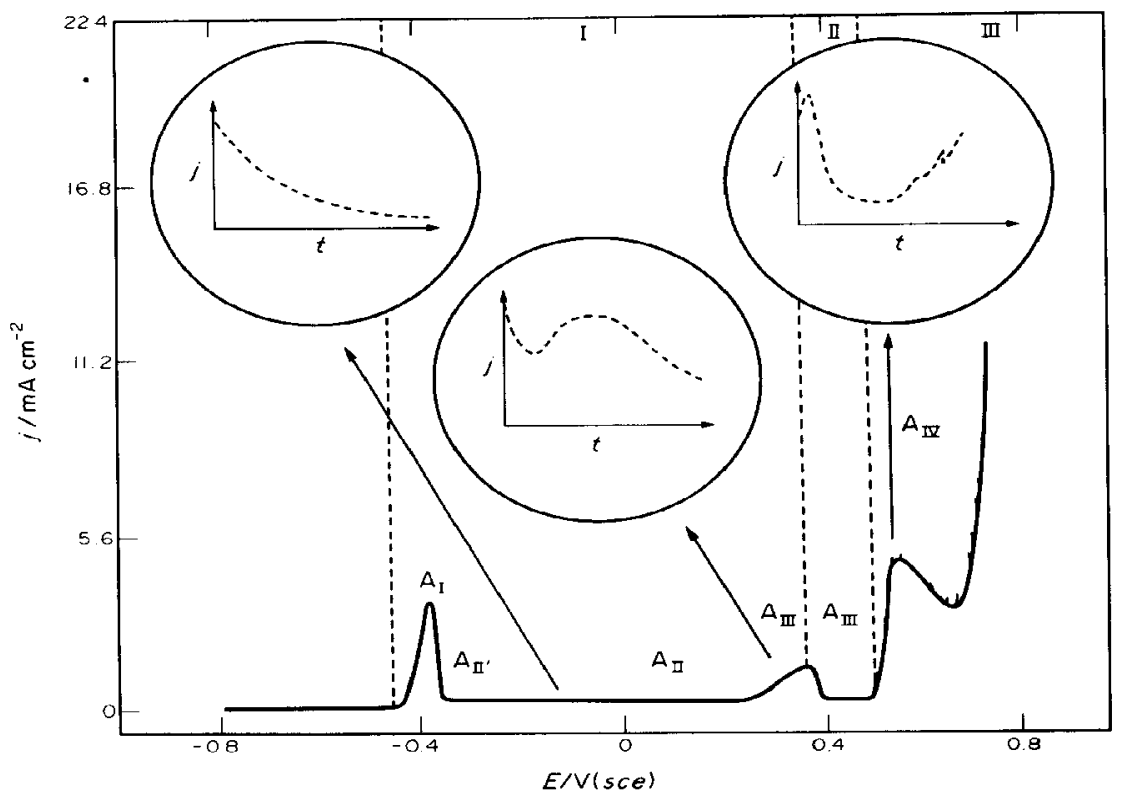

Fig. 1. Polarization curve of copper recorded at $0.002 \mathrm{~V} \mathrm{~s}^{-1}$ in the borax buffer containing $0.3 \mathrm{M} \mathrm{KSCN}$. The diagrams in the circles show the shape of the current transients recorded in the different potential regions. Potential regions I, II and III are indicated in the upper part.

centration. Region III extends beyond the passivity breakdown potential $\left(E_{b}\right)$ which is associated with pitting corrosion of copper. As recently reported, during pitting the electrode is partially covered by a black layer of cupric thiocyanate. As the composition of this layer changes from cupric into cuprous thiocyanate a certain inhibition of copper pitting is noticed (AIV). The detailed analysis of the voltammetric response of copper in potassium thiocyanate solutions was already reported in part I[6]. As the concentration of the latter increases, $E_{b}$ moves towards lower potentials, and occasionally it falls within the potential range of peaks AIII/AIII'.

\section{Potentiostatic current transients}

Current transients under a constant potential step $\left(E_{\mathrm{s}}\right)$ in $0.3 \mathrm{M}$ potassium thiocyanate were obtained by applying to the copper electrode the following pretreatment. Firstly, the potential was stepped for $90 \mathrm{~s}$ to $-1.00 \mathrm{~V}$, then to $-0.3 \mathrm{~V}$ for $90 \mathrm{~s}$ to form a prepassive CuSCN layer, and finally to $E_{s}$ to record the current transient.

For $E_{s}<E_{\text {AllI }}$ only a decrcasing transient related to the completion of the passive layer is observed (Fig. 2, plots $a, b)$ so that the integrated charge increases according to $E_{s}$. For $t>2 \mathrm{~s}$ the $j v s t^{-1 / 2}$ plot shows a linear dependence. At $E_{s} \simeq E_{\text {AIII }}$ the current initially decreases, later it increases to attain a maximum value, $I_{M}$ at $t_{M}$, and finally it again decreases. Likewise, as $E_{s}$ is set more positive, $I_{M}$ increases and $t_{M}$ decreases (Fig. 2 , plots c, d). The integral charge involved up to $I_{M}$ is related to that of peak AIII in the voltammogram. Furthermore, at longer times the current transients exhibit another small current maximum (not shown in

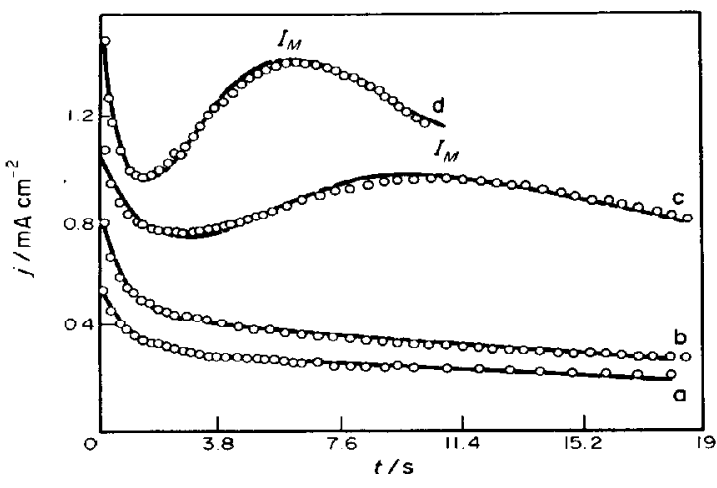

Fig. 2. Current transients at constant potential recorded in buffer $+0.3 \mathrm{M} \mathrm{KSCN}$ after applying to the electrode the following pretreatment: $E_{c}=-1.00 \mathrm{~V}, t_{c}=90 \mathrm{~s}, E_{a}=-0.3 \mathrm{~V}$, $t_{a}=90 \mathrm{~s}$, and later potential stepped to (a) $E_{s}=0.2 \mathrm{~V}$, (b) $E_{\mathrm{s}}=0.30 \mathrm{~V}$, (c) $E_{\mathrm{s}}=0.36 \mathrm{~V}$, (d) $E_{\mathrm{s}}=0.40 \mathrm{~V}$. (o) Experimental data, (-) calculation using equations (2), (5) (plots a and b), and (2), (14) (plots c and d).

Fig. 2), which correlates to voltammetric peak AIII'. Similar experiments were run with the rrde $\left(E_{r}=\right.$ $-0.80 \mathrm{~V}$ ) by forming the passive layer at the disc electrode and following the formation of soluble copper species at the ring electrode held at a constant potential. The electroformation of soluble copper species operates already from $t=0$ onwards and it reaches a maximum amount at the time $t_{M}$ (Fig. 3). It appears that the current transient at the disc is formed of two different contributions, one in the short time range which decreases with time, and another in the 


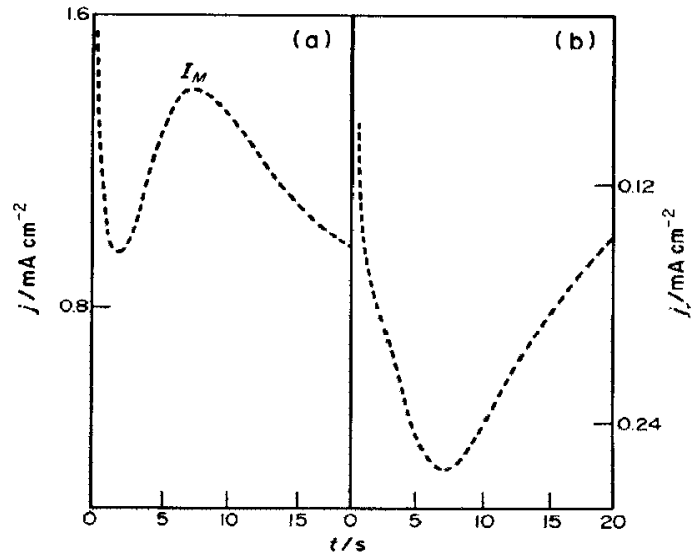

Fig. 3. (a) Current transient at the disc electrode $\left(E_{s}=0.325 \mathrm{~V}\right)$. The electrode was pretreated as described in the legend of Fig. 2. (b) Current transient at the ring electrode $\left(E_{r}=-0.80\right)$. Electrolyte: borax buffer $+0.3 \mathrm{M} \mathrm{KSCN}, w=250 \mathrm{rpm}$.

long time range which increases up to maximum. Apparently, those processes associated with the second contribution are the only ones impliying the formation of soluble copper species.

For $E_{s}>E_{\text {AII }}$ the current transients exhibit the same features already described for $E_{s} \simeq E_{\text {AIII }}$ although in this case $t_{M}$ becomes very small and $I_{M}$ results considerably increased. The integrated charge up to $I_{M}$ becomes practically independent of $E_{s}$. Otherwise, when $E_{s}>E_{b}$ a second current maximum $\left(I_{M}^{\prime}\right)$ is observed at $t_{M}^{\prime}$ (Fig. 4). The faradaic processes related to $I_{M}^{\prime}$ involve an induction time $\left(t_{i}\right)$. This portion of the current transient also exhibits a considerable noise. Both $t_{M}^{*}$ and $t_{i}$ decrease as $E_{s}$ increases. The charge associated with $I_{M}^{\prime}$ correlates to the charge of the voltammetric peak AIV. For the latter current oscillations are also observed in the slow sweep rate voltammogram (Fig. 1).
For $E_{s}>E_{b y}$ the rapid initial decrease of the pitting current is accompanied by patches of the electrode surface changing in colour from black into white. This corresponds to a partial inhibition of the pitting process so that only certain areas of the electrode remain active as revealed by the slow current increase observed at longer times.

To verify the ability of thiocyanate ions to break the passive film formed in the absence of potassium thiocyanate the oxide film was previously formed in the borax buffer, at $0.80 \mathrm{~V}$, during $400 \mathrm{~s}$. Immediately afterwards, $5 \mathrm{ml}$ of borax + potassium thiocyanate concentrated solution was added to the electrolyte. The addition of potassium thiocyanate changes the continuously decreasing transient into a sudden current increase associated with uneven oscillations (Fig. 5). This response confirms that $I_{M}^{\prime}$ is exclusively related to the pitting process, and that in this case the processes related to $I_{M}$ are cancelled because the passive layer has been formed previously to potassium thiocyanate addition. It is also observed that the initial black spots on the electrode $\left[\mathrm{Cu}(\mathrm{SCN})_{2}\right]$ are partially converted into white spots (CuSCN), probably through the formation of soluble Cu(SCN) 3 species.

\section{DISCUSSION}

The voltammetric response of copper in borate-boric acid buffer containing potassium thiocyanate involves the appearance of different new phases at the electrode surface along the potential scan. In region $I\left(E_{\mathrm{Al}}<E_{s}<E_{\mathrm{AIII}}\right)$ cuprous thiocyanate, hydrous cuprous oxide and hydrous cupric oxide are successively formed as the applied potential is increased. Once the composite anodic layer in region $I$ is completed, a passive region extending from $E_{\mathrm{AII}}$ to a potential close to $E_{b}$ (region II) can be assigned. Finally, when the applied potential exceeds $E_{b}$, the breakdown of passivity caused by the presence of thiocyanate ions is observed (region III). Potentiostatic current transients run in the three voltammetric regions correlate to data earlier reported

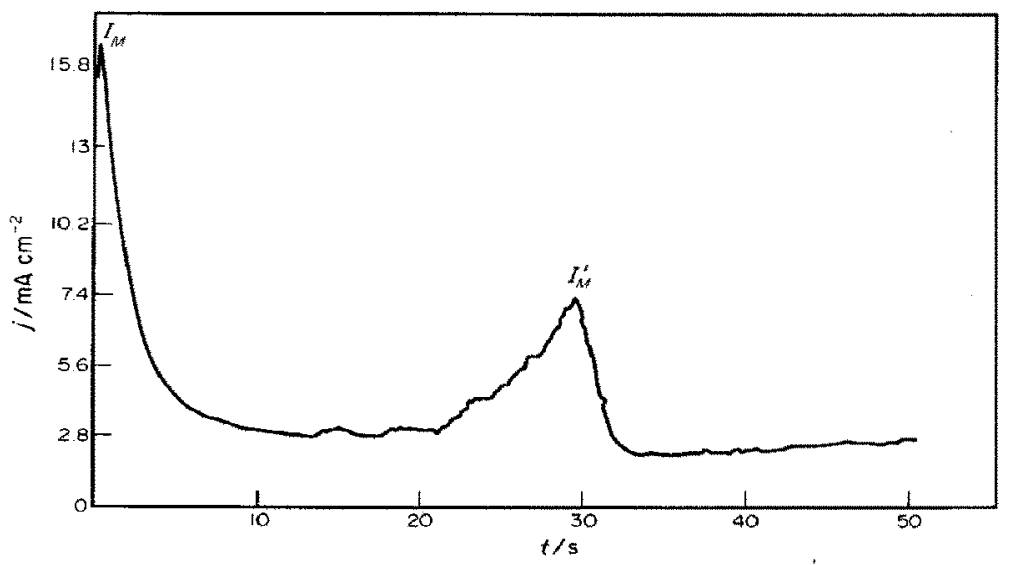

Fig. 4. Current transient at constant potential $E_{s}>E_{b}$ in the borax buffer $+0.5 \mathrm{M} \mathrm{KSCN}\left(E_{x}=0.70 \mathrm{~V}\right)$. The electrode pretreatment is the same described for Fig. 2. 


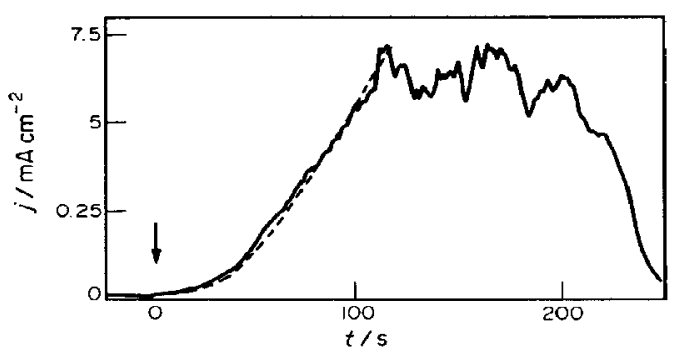

Fig. 5. Current transient at constant potential $\left(E_{s}=0.80 \mathrm{~V}\right)$ for a prepassivated copper electrode $(t=400 \mathrm{~s})$ after addition of $5 \mathrm{ml}$ borax buffer + concentrated KSCN up to $0.3 \mathrm{M}$ KSCN. The arrow indicates addition of KSCN solution. ' $I$ 'he dashed line is calculated with equation (22).

[7-9] and offer the posibilities of further understanding some mechanistic aspects of the entire process.

\section{Analysis of current transients in region $I$}

Potentiostatic current transients in the $\mathrm{F}_{\mathrm{AI}}<\mathrm{F}_{\mathrm{s}}$ $<\mathrm{E}_{\mathrm{AlII}}$ range. In region $\mathrm{I}$ most of the charge involved in voltammetric peak $A I$ is related to the formation of a cuprous thiocyanate layer presumably in contact with the base metal surface, and a small fraction of it corresponds to the electroformation of soluble copper species. This is supported by EDAX, electron microprobe analysis and SEM observations of the electrode surface after anodizing in this potential range showing a thick and crystalline porous CuSCN film [6] as already reported in part $I$. The charge density for cuprous thiocyanate formation is about $\mathbf{7 0}$ $\pm 7 \mathrm{mCcm}^{-2}$, whereas the complementary electroreduction charge is about $50 \pm 5 \mathrm{mC} \mathrm{cm}^{-2}$ [6]. From the latter, providing that $M=121 \mathrm{~g} \mathrm{~mol}^{-1}, z=1, \rho$ $=2.8 \mathrm{~g} \mathrm{~cm}^{-3}$ and a roughness factor equal to 1 , the minimum average thickness of the cuprous thiocyanate layer results about $3 \times 10^{-5} \mathrm{~cm}$. The linear dependence of both the potential and height of peak AI on $v^{1 / 2}$, and the independence of the peak height on the solution stirring indicate that the kinetics of the cuprous thiocyanate electroformation is mainly under ohmic control[10].

At $E_{s}>E_{\mathrm{AI}}$ the continuously decreasing current transients are related to the electroformation of a hydrous cuprous oxide layer underneath the porous cuprous thiocyanate layer (Fig. 2), and in the corresponding potential range copper electrodissolution through the composite anodic film takes place as detected through the rrde technique[6]. Therefore, the current transient $[j(t)]$ in this case should involve the sum of two contributions, namely, one related to the electroformation of the hydrous cuprous oxide film $\left[j_{p}(t)\right]$ and another assigned to the electrodissolution of base copper through the composite film $\left[j_{\mathrm{CI}}(t)\right]$. Thus:

$$
j_{T}(t)=j_{p}(t)+j_{\mathrm{CI}}(t)
$$

Both current components involve ion transport through the film formed during the prepassivation period at $-0.30 \mathrm{~V}$ prior to the potential step. Taking into account film structure and thickness as reported in part I, a high-field mechanism can be disregarded and on the basis of the corresponding current transient mathematical fitting ion diffusion through the CuSCN layer appears as the more likely mechanism. The electroformation of the hydrous cuprous oxide layer can be interpreted through an instantaneous nucleation and 2-D growth mechanism under diffusion control. Then, the expression for $j_{p}(t)$ is [11]:

$$
j_{p}(t)=p_{1} \exp \left(-p_{2} t\right)
$$

where

$$
\begin{aligned}
& p_{1}=q \pi K_{e} D N_{0} \\
& p_{2}=\pi K_{e} D N_{0}
\end{aligned}
$$

$q$ is the charge density related to the completion of the cuprous oxide layer, $K_{e}$ is a proportionality constant, $N_{0}$ is the number of nucleation sites and $D$ is the diffusion coefficient of the reacting species, presumably water molecular diffusing from the solution side through the cuprous thiocyanate layer.

On the other hand, the corrosion of copper through the composite anodic layer produces the electrodissolution of the base metal. Recently, this type of corrosion process has been also interpreted in terms of a nucleation and growth mechanism[7]. Hence, in the present case the corrosion of copper underlying the composite anodic layer can be explained by assuming an instantaneous nucleation and 3-D growth of holes under diffusion control. The electrodissolution of copper likely implies the diffusion of copper ions through the composite anodic layer as no dependence of $j$ on electrode rotation is observed [6]. Then, the value of $j_{\mathrm{Cl}}(t)$ derived for the proposed model can be written as follows[12]:

$$
\begin{gathered}
j_{\mathrm{CI}}(t)=\frac{p_{3}}{\sqrt{t}}\left[1.0-\exp \left(-p_{4} t\right)\right] \\
p_{3}=z F\left(D^{\prime}\right)^{1 / 2} \Delta c^{\prime} / \pi^{1 / 2} \\
p_{4}=\pi K_{e} D^{\prime} N_{0}
\end{gathered}
$$

where $\Delta c^{\prime}$ and $D^{\prime}$ are the concentration gradient in the film normal to the electrode surface and the diffusion coefficient of metallic ions, respectively. Current transients shown in Fig. 2 can be reproduced within $5 \%$ from equation (2) and (5) with the parameters assembled in Table 1 .

From these runs where the cuprous thiocyanate layer has been previously formed it is possible to estimate the average thickness $(h)$ of the inner passivating layer formed at a constant potential in region $I$ by using the following relationship:

$$
h=M p_{1} / z F \rho p_{2} \text {. }
$$

On the assumption that the inner passivating layer formed at $E_{s}$ is mainly constituted of cuprous oxide $\left(M=143 \mathrm{~g} \mathrm{~mol}^{-1}, \rho=6.0 \mathrm{~g} \mathrm{~cm}^{-3}, z=1\right)$, it results $h$ $=9 \AA$ for $E_{s}=0.1 \mathrm{~V}$, and $h=18 \AA$ for $E_{s}=0.3 \mathrm{~V}$. These figures are in good agreement with previously reported data in plain buffer solution[9]. Therefore, from these results one concludes that the anodic layer electroformed in region I comprises an inner thin hydrous cuprous oxide layer and an outer thick cuprous thiocyanate layer. Equation (5) predicts that when $p_{4} t \rightarrow \infty$, lineal $j$ ss $t^{-1 / 2}$ relationships must be obtained in agreement with the experimental observa- 
Table 1. Adjusting parameters for the current transients

\begin{tabular}{cccccccc}
\hline Fig. & $E(\mathrm{~V})$ & $p_{1}\left(\mathrm{~mA} \mathrm{~cm}^{-2}\right)$ & $p_{2}\left(\mathrm{~s}^{-1}\right)$ & $p_{3}\left(\mathrm{~mA} \mathrm{~s}^{1 / 2} \mathrm{~cm}^{-2}\right)$ & $p_{4}\left(\mathrm{~s}^{-1}\right)$ & $p_{5}\left(\mathrm{~mA} \mathrm{~cm}^{-2}\right)$ & $p_{6}\left(\mathrm{~s}^{-2}\right)$ \\
\hline- & 0.10 & 0.211 & 0.636 & 0.614 & 0.213 & - & - \\
2 & 0.20 & 0.520 & 0.923 & 0.832 & 0.254 & - & - \\
2 & 0.30 & 0.774 & 1.02 & 1.15 & 0.282 & - & - \\
2 & 0.36 & 1.09 & 0.327 & - & - & 3.42 & 0.0212 \\
2 & 0.40 & 1.65 & 0.740 & - & - & 3.83 & 0.061 \\
\hline
\end{tabular}

tions. Otherwise, the average value of $\Delta c^{\prime}$ can be estimated for simple diffusion geometry from $p_{3}$ through equation (6). By taking $D^{\prime}=1 \times 10^{-13}$ $\mathrm{cm}^{2} \mathrm{~s}^{-1}[13]$, values of $\Delta c^{\prime}$ comprised between 0.036 and $0.067 \mathrm{molcm}^{-3}$ are obtained. Likewise, from the value of $\Delta c^{\prime}$, the limiting current $\left(j_{L}\right)$ observed between peaks AI and AIII can be calculated from the relationship:

$$
j_{L}=z F D^{\prime} \Delta c^{\prime} / h^{\prime}
$$

where $h^{\prime}$ corresponds to the thickness of the diffusion layer which is taken as the entire thickness of the composite anodic layer. Thus, for $z=1, D^{\prime}=1$ $\times 10^{-13} \mathrm{~cm}^{2} \mathrm{~s}^{-1}, \Delta c^{\prime}=0.03 \mathrm{~mol} \mathrm{~cm}{ }^{-3}$ and $h^{\prime}-3$ $\times 10^{-5} \mathrm{~cm}$, it results $j_{L}=1.2 \times 10^{-5} \mathrm{~A} \mathrm{~cm}^{-2}$. This figure is close to the limiting current $j_{L}=1.4 \times 10^{-5}$ $\mathrm{A} \mathrm{cm}^{-2}$ ) recorded at $0.2 \mathrm{mV} \mathrm{s}^{-1}$.

Potentiostatic current transient at $\mathrm{E}_{\mathrm{s}} \simeq \mathrm{E}_{\mathrm{AlI}}$. For $E_{s} \simeq E_{\mathrm{AIII}}$ both $\mathrm{Cu}(\mathrm{I})$ species as well as base copper are electrooxidized yielding cupric oxide and soluble $\mathrm{Cu}$ (II). The formation of soluble $\mathrm{Cu}$ (II) is confirmed through the rrde technique and it occurs from either base copper or $\mathrm{Cu}(\mathrm{I})$ or by complex formation from $\mathrm{Cu(II)}$ species in the solid phase, according to the following reactions, respectively:

$$
\begin{gathered}
\mathrm{Cu} \rightleftharpoons \mathrm{Cu}^{2+}+2 e^{-} \\
\mathrm{Cu}^{+}(\text {solid state }) \rightleftharpoons \mathrm{Cu}^{2+}+e^{-} \\
\mathrm{Cu}^{2+}+3 \mathrm{SCN}^{-} \rightleftharpoons \mathrm{Cu}(\mathrm{SCN})_{3}^{-} .
\end{gathered}
$$

Reactions (11) and (12) result in the electrodissolution of the outer thick CuSCN layer leading to hole formation as detected by SEM [6] and it explains that only a small fraction of the overall anodic charge of peak AIII appears in the complementary electroreduction profile. Accordingly, the process occurring at $E_{s}$ $\simeq E_{\text {AIII }}$ becomes dependent on the hydrodynamics of the system.

For $E_{s} \simeq E_{\mathrm{AIII}}$, the overall current transient $j_{T}(t)$ can be considered as the sum of the contributions $j_{p}(t)$, $j_{\mathrm{CI}}(t)$ and $j_{\mathrm{CII}}(t)$ so that

$$
j_{T}(t)=j_{p}(t)+j_{\mathrm{Cl}}(t)+j_{\mathrm{CII}}(t)
$$

$j_{p}(t)$ can be expressed by equation (2) and it is related to the passive layer formation in this potential range. This layer approaches the inner $\mathrm{Cu}_{2} \mathrm{O}$ /outer hydrous $\mathrm{CuO}$ structure. $j_{C I}(t)$ is given by equation (5) and it is associated with the copper electrodissolution through the composite layer. $j_{\mathrm{CII}}(t)$ represents the dissolution of the outer CuSCN layer as given by reactions (11) and (12) and it determines the current maximum $I_{M}$ at $t_{M}$. This electrodissolution process can be envisaged as a progressive nucleation and 3-D growth of holes under diffusion control. Then, the corresponding equation for $j_{\mathrm{CII}}(t)$ is:

$$
\begin{gathered}
j_{\mathrm{CII}}(t)=\frac{p_{5}}{\sqrt{ } t}\left[1.0-\exp \left(-p_{6} t^{2}\right)\right] \\
p_{5}=z F\left(D^{\prime \prime}\right)^{1 / 2} \Delta c^{\prime \prime} / \pi^{1 / 2} \\
p_{6}=D^{\prime \prime} K_{\mathrm{e}}^{\prime \prime} A N_{0} \pi .
\end{gathered}
$$

$D^{\prime \prime}$ and $\Delta c^{\prime \prime}$ are the diffusion coefficient in solution and the concentration gradient of $\mathrm{Cu}(\mathrm{SCN})_{3}^{-}$ions normal to the electrode surface and $A N_{0}$ is the nucleation rate of holes. Considering that $j_{\mathrm{CI}} \ll j_{\mathrm{Cl}}$, equation (13) becomes:

$$
j=j_{\mathrm{p}}+j_{\mathrm{CII}}
$$

Using equation (2) and (14) the current transients (Fig. 2) can be reproduced with the parameters assembled in Table 2. On the assumption that the electrodissolution charge is mainly related to hole formation in the cuprous thiocyanate outer layer [equation(11)], $\Delta c^{\prime \prime}$ can be estimated from the value of $p_{5}$. Taking $D^{\prime \prime}$ $=10^{-5} \mathrm{~cm}^{2} \mathrm{~s}^{-1}, z=1$ and $p_{5}=3.42 \times 10^{-3} \mathrm{~A} \mathrm{seg}^{1 / 2}$ $\mathrm{cm}^{-2}$ it results $\Delta c^{\prime \prime}=10^{-5} \mathrm{~mol} \mathrm{~cm}{ }^{-3}$. The value $A N_{0}$ can be estimated from $p_{6}$ providing that the value of

\begin{tabular}{|c|c|c|c|c|c|c|c|c|c|c|}
\hline Fig. & $E(\mathbf{V})$ & $p_{1}\left(\mathrm{~mA} \mathrm{~cm} \mathrm{~cm}^{-2}\right)$ & $p_{2}\left(s^{-1}\right)$ & $p_{7}\left(\mathrm{~mA} \mathrm{~cm}^{-2}\right)$ & $p_{8}\left(s^{-2}\right)$ & $p_{9}\left(\mathrm{~mA} \mathrm{~cm}^{-2}\right)$ & $p_{10}\left(\mathrm{~s}^{-2}\right)$ & $p_{11}\left(\mathrm{~mA} \mathrm{~cm} \mathrm{~cm}^{-2}\right)$ & $p_{12}\left(s^{-2}\right)$ & $t_{i}(\mathrm{~s})$ \\
\hline $6 a$ & 0.65 & 9.65 & 0.329 & 18.0 & 0.802 & 1.72 & 0.320 & - & - & - \\
\hline $6 \mathrm{~b}$ & 0.70 & 10.38 & 0.438 & 20.5 & 1.13 & 2.43 & 0.165 & - & - & - \\
\hline 7 & 0.65 & 9.65 & 0.329 & - & - & 1.72 & - & 14.0 & 0.0033 & 27.1 \\
\hline 7 & 0.70 & 10.4 & 0.438 & - & - & 2.43 & - & 25.0 & 0.0025 & 18.4 \\
\hline 7 & 0.75 & 11.9 & 0.510 & - & - & 2.86 & - & 35.0 & 0.0055 & 9.79 \\
\hline 5 & 0.80 & - & - & - & - & - & - & 21.0 & 0.0003 & 10.1 \\
\hline
\end{tabular}
$K_{e}^{\prime \prime}$ can be calculated from the relationship[12]:

$$
K_{e}^{\prime \prime}=\frac{4}{3}\left(\frac{8 \pi \Delta c^{\prime \prime} M}{\rho}\right)^{1 / 2} .
$$

For $\Delta c^{\prime \prime}=10^{-5} \mathrm{molcm}^{-3}, M=121 \mathrm{~g} \mathrm{~mol}^{-1}$ and $\rho$ $=2.8 \mathrm{~g} \mathrm{~cm}^{-3}, K_{e}^{\prime \prime}=0.1389$. From equation (16) it results for $E_{s}=0.36 \mathrm{~V}, A N_{0}=4.3 \times 10^{3} \mathrm{~cm}^{-2} \mathrm{~s}^{-1}$ and for $E_{s}=0.40 \mathrm{~V}, A N_{0}=1.36 \times 10^{4} \mathrm{~cm}^{-2} \mathrm{~s}^{-1}$.

Table 2. Adjusting parameters for the current transients 
Analysis of current transients in region $I I I$

In region $\operatorname{III}\left(E_{s}>E_{b}\right)$ the transients exhibit an initial falling current followed by the maximum $I_{M}$ which are related with the processes described previously although the sharp $I_{M}$ peak is unexpected for a 3-D growth under diffusion control [equation(14)]. Furthermore, when $t_{M}$ is exceeded a constant current region is observed and afterwards the current increases to a second noisy maximum $\left(I_{M}^{\prime}\right)$ at the time $t_{M}^{\prime}$. Later it decreases suddenly, and finally it increases again but very slowly (Fig. 4). These current contributions are related to the pitting of copper and the nucleation and growth of cupric thiocyanate. Thus, for $t<t_{M}^{\prime}$, the total current can be represented as the sum of four contributions:

$$
j_{T}(t)=j_{p}(t)+j_{\mathrm{CIII}}(t)+j_{\mathrm{CIV}}(t)+j_{s}(t)
$$

where $j_{p}(t)$ is related to the processes occurring during the initial current decrease and is given by equation (2); $j_{\mathrm{CIII}}(t)$ is associated with those processes responsible for the current maximum $I_{M}$, and in this potential range it can be represented as a progressive nucleation and 2-D growth of holes under diffusion control[14]. The terms $j_{C I V}(t)$ and $j_{s}(t)$ are related to pitting and are assigned to an instantaneous nucleation and conical growth under charge transfer control[15]. The corresponding equations for $j_{\mathrm{CIII}}(t), j_{\mathrm{CIV}}(t)$ and $j_{s}(t)$ are given by:

$$
\begin{gathered}
j_{\mathrm{CIII}}=p_{7} t \exp \left(-p_{8} t^{2}\right) \\
j_{\mathrm{CIV}}=p_{9}\left[1.0-\exp \left(-p_{10} t^{2}\right)\right] \\
j_{s}=p_{11}\left\{1.0-\exp \left[-p_{12}\left(t-t_{i}\right)^{2}\right]\right\} \\
p_{7}=q \pi k D^{\prime \prime} N_{0}^{\prime} A^{\prime} \\
p_{8}=\pi k D^{\prime \prime} N_{0}^{\prime} A^{\prime} / 2 .
\end{gathered}
$$

$k$ is a proportionality constant and $A N_{0}^{\prime}$ is the nucleation rate.

$$
\begin{gathered}
p_{9}=p_{11}=z F k_{3 c}^{\prime} \\
p_{10}=p_{12}=M_{s}^{2} N_{0 s} k_{3 c}^{2} \pi / \rho_{s}^{2} .
\end{gathered}
$$

$k_{3 c}$ and $k_{3 c}^{\prime}$ are formal rate constants for the growth parallel and perpendicular to the ideal surface electrode plane, respectively. $M_{s}$ is the molecular weight of cupric thiocyanate whose density is $\rho_{s}, j_{\mathrm{CIV}}$ and $j_{s}$ are related to the $\mathrm{Cu}(\mathrm{SCN})_{2} /$ oxide and $\mathrm{Cu}(\mathrm{SCN})_{2} / \mathrm{Cu}$ interfaces, respectively. For simulating the current transients different time scales must be considered to handle simplified forms of the equation (19). Thus, for $0<t<t_{i}$, the contribution of $j_{s}$ becomes negligible, so that:

$$
j_{T}(t)=j_{p}(t)+j_{\mathrm{CIII}}(t)+j_{\mathrm{CIV}}(t) .
$$

Therefore, the initial part of the current transient shown in Fig. 4 can be reproduced using the adjusting parameters assembled in Table 2 (Fig. 6).

Current transients recorded at $0.4 \mathrm{~V}<E_{\mathrm{s}}<0.6 \mathrm{~V}$ present a behaviour which is intermediate between that observed for $E_{s}<0.4 \mathrm{~V}$ (3-D nucleation and growth) and that for $E_{s} \geqslant 0.6 \mathrm{~V}$ (2-D nucleation and growth). In these cases, no simple model can be used for a satisfactory curve fitting.

Otherwise, for $3 \mathrm{~s}<t<t_{M}^{\prime}, j_{\mathrm{CIII}}(t)$ approaches zero and $j_{\mathrm{CIV}} \simeq p_{9}$. Thus:

$$
j_{T}(t)=j_{p}(t)+p_{9}+j_{s}
$$

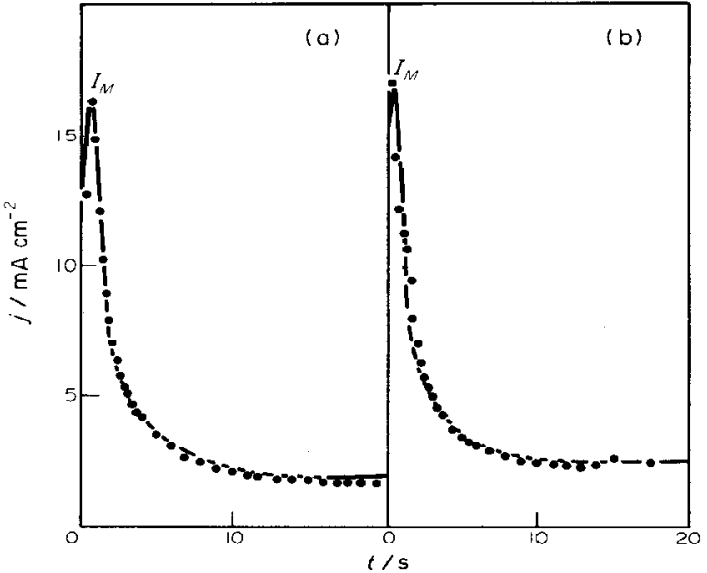

Fig. 6. Initial part of current transients $\left(0 \leqslant t \leqslant t_{i}\right)$ at $E_{\mathrm{s}}>E_{b}$ in the borax buffer $+0.5 \mathrm{M}$ KSCN. (0) Experimental data, $(-)$ calculation using equations (2), (19), (20), (a) $E_{s}=0.65 \mathrm{~V}$; (b) $E_{s}=0.70 \mathrm{~V}$.

In this case, the current transients can be reproduced by using equations (2), (22) and (28) with the parameters assembled in Table 2 (Fig. 7). As $E_{s}$ increases both $p_{1}$ and $p_{2}$ increase but the $p_{1} / p_{2}$ ratio $(q)$ remains practically constant. Therefore, as $E_{s}$ moves towards more positive potential values, $p_{9}, p_{11}$ and $p_{12}$ increase and $t_{i}$ decreases. The values of $t_{i}$ satisfy linear $\log t_{i} v \mathrm{~s}$ $1 /\left(E_{s}^{-} E_{b}\right)$ and $\log t_{i} v s\left(E_{s}-E_{b}\right)$ relationships, similar to those already reported in the literature $[16,17]$. As the pitted area of the electrodes is a small fraction of the total area (about $1 \%$ ), $p_{11}$ should be related to current densities in the order of $\mathrm{A} \mathrm{cm}^{-2}$. However it is known that pitting measurements are poorly reproducible[18] and the scatter should affect the adjusting parameters so that its physical meaning can be questioned. This is specially true at $E_{s} \simeq E_{b}$ but as the $\left(E_{\mathrm{s}}\right.$ $-E_{b}$ ) value becomes larger the scatter is drastically reduced and well-defined transients are recorded. The influence of $E_{s}$ on the pitting transients and the transition from a stochastic model to a deterministic one to describe the pitting kinetics need to be examined

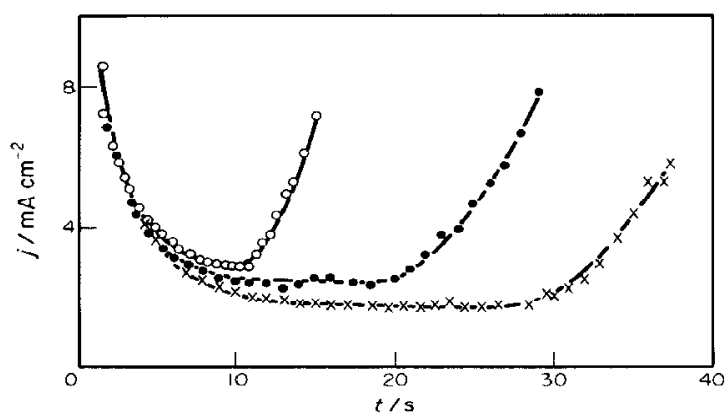

Fig. 7. Current transients $\left(3 \mathrm{~s}<t<t_{M}^{\prime}\right)$ recorded at $E_{s}>E_{b}$ in the borax buffer $+0.5 \mathrm{M}$ KSCN. ( $\longrightarrow$ Calculation using equations (2), (22), (28). ( $\times$ ) $0.65 \mathrm{~V}$; (-) $0.70 \mathrm{~V}$; (o) $0.75 \mathrm{~V}$, experimental data. 

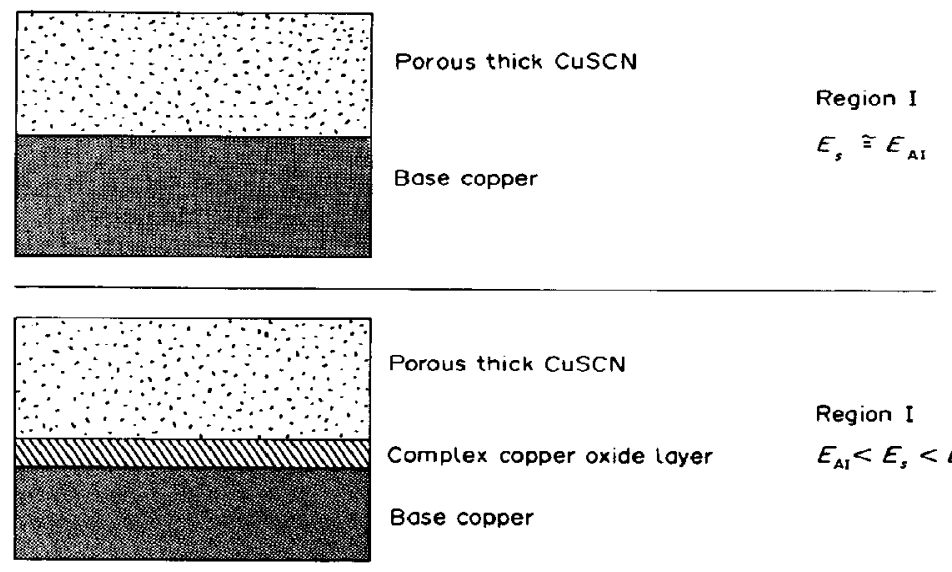

Porous thick CuSCN

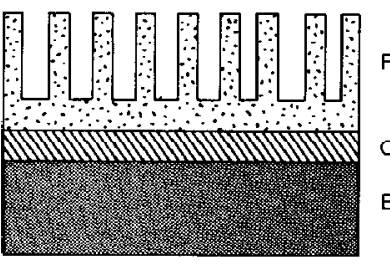

Porous thick CuSCN

\section{Region I}

$E_{s} \cong E_{\text {AIII }}$

Complex copper oxıde tayer

Bose copper

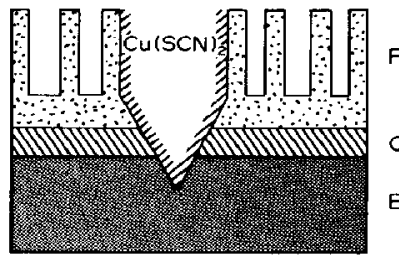

Porous thick CuSCN

Complex copper oxide tayer

Region III

$E_{s}>E_{0}$

Bose copper

Fig. 8. Schematic representation of the copper anodic layer structure associated with the different potential regions.

further[19]. The validity of equation (22) to describe pit growth was confirmed in separate experiments where other current contributions can be neglected. Thus, for the current transients resulting with prepassivated electrodes one should expect that $j_{p} \rightarrow 0$ and $j_{\text {CIV }}$ becomes negligible, so that equation (27) becomes:

$$
j_{T}=j_{s} \text {. }
$$

The current transient using prepassivated electrodes (Fig. 5) are theretore fitted using equation (22) with parameters given in Table 2 .

Finally, the sudden decrease in current after time $t_{M}^{\prime}$ (Fig. 5) can be attributed to the partial blocking of the pitted areas by a porous CuSCN layer and precipitated compounds formed through the following reactions:

$$
\begin{gathered}
(\mathrm{SCN})_{2} \mathrm{Cu}+\mathrm{SCN}^{-} \rightarrow(\mathrm{SCN})_{3} \mathrm{Cu}^{-} \\
(\mathrm{SCN})_{3} \mathrm{Cu}^{-} \rightleftharpoons \mathrm{CuSCN}+(\mathrm{SCN})_{2}+e^{-}
\end{gathered}
$$

When this situation is achieved the current increases slowly with time due to the increase in roughening of the corroding copper surface mostly covered by corrosion products.

In conclusion, the corrosion and passivation of $\mathrm{Cu}$ in borate-boric acid buffer containing thiocyanate ions entails the participation of different electrochemical processes, each one of them being most relevant within a certain potential window. A schematic representation of the copper anodic layer structures associated with the different potential regions is depicted in Fig. 8. The extension of the nucleation and growth mechanism to the formation and disappearance of the different phases allows a reasonable interpretation of the kinetics of the complex processes in the entire potential range associated with the thermodynamic stability of bulk water.

Acknowledgement-This research project is financially supported by the Universidad Nacional de La Plata, the Consejo Nacional de Investigaciones Científicas y Técnicas and the Comisión de Investigacioncs Científicas de la Provincia de Buenos Aires. 


\section{REFERENCES}

1. F. M. Al-Kharafi and Y. A. El-Tantawy, Corros. Sci. 22, 1 (1982)

2. G. Faita, G. Fiori and D. Salvadore, Corros. Sci. 15, 383 (1975).

3. M. R. G. de Chialvo, R. C. Salvarezza, D. V. Vasquez Moll and A. J. Arvia, Electrochim. Acta 30, 1011 (1985).

4. H. H. Strehblow and B. Titze, Electrochim. Acta, 25, 339 (1980).

5. J. R. Galvele, J. electrochem. Soc. 123, 464 (1976)

6. M. G. Figueroa, R. C. Salvarezza and A. J. Arvia, Electrochim. Acta, 31, 671 (1986).

7. D. V. Vasquez Mool, R. C. Salvarezza, H. A. Videla and A. J. Arvia, J. electrochem. Soc. 132, 754 (1985).

8. D. V. Vasquez Moll, R. C. Salvarezza, H. A. Videla and A. J. Arvia, Corros. Sci. 24, 751 (1984).

9. M. R. G. de Chialvo, D. V. Vasquez Moll, R. C Salvarezza and A. J. Arvia, Electrochim. Acta 30, 1501 (1985).
10. A. J. Calandra, N. R. de Tacconi, R. Pereiro and A. J. Arvia, Electrochim. Acta 19, 901 (1974).

11. W. Davison and J. A. Harrison, J. electroanal. Chem. 44, 213 (1973).

12. B. Scharifker and G. Hill, Electrochim. Acta 28, 879 (1983).

13. Z. Szklarska-Smialowska and W. Kozlowski, J. electrochem. Soc. 131, 234 (1984).

14. H. R. Thirsk and J. Harrison, A Guide to the Study of the Electrode Kinetics. Academic Press, London (1972).

15. M. Y. Abyaneh and M. Fleichmann, Electrochim. Acto 27, 1513 (1982).

16. K. E. Heusler and L. Fischer, Werkst. Korros. 109, 679 (1976).

17. T. Okada, J. electrochem. Soc. 131, 241 (1984).

18. D. E. Williams, C. Westcott and M. Fleischmann, $I$. electrochem. Soc. 132, 1797 (1985).

19. R. C. Salvarezza, N. de Cristoofaro, C. Pallotta and A. J. Arvia, Electrochim. Acta, submitted. 